\title{
Research on Embedded Project Risk Assessment and Control
}

\author{
Chunmei Ji, Nengjun Ben \\ Yancheng Vocational Institute of Industry Technology; Yancheng Jiangsu 225005 China.
}

\begin{abstract}
With the development of embedded technology, the application of embedded products is dazzling. Whether the system project can guarantee the completion of quality has become a problem to be solved at present. Due to the uncertainty and uniqueness of the embedded development process, how to assess the requirement risk of this project has become a difficult problem. In the development cycle, the time for finding the problem is directly proportional to the cost of repairing the problem. The earlier the problem is discovered, the lower the cost of repair, and the requirement is just the initial stage of project implementation. This article mainly introduces the characteristics of embedded development and the causes of the requirement risk. According to the requirement risk of the embedded project in the development environment, it leads to the delay of the project, and constructs the index system principle of the requirement risk for the embedded project.
\end{abstract}

Key words: embedded project; risk assessment; requirement risk; Indicator system principle.

\section{INTRODUCTION}

The work flow path of Embedded project [1] is complicated, any problem with one process can cause emergencies in other processes, consequently, the program delays. Therefore, we cannot assess the project's requirement risk [2] adequately without making full sense of the requirement and scope of the project. According to the survey, Forty-five percent of the projects fail because of improper requirements. Therefore, making requirement reasonably is the key factor to ensure the quality and punctuality of the project. Every step to making requirement is full of risks [3]. At present, the evaluation method of risk is very simple, which cannot assess the risk of the requirement stage effectively. which leads to the weak embedded project, so that the development cycle is delayed. This leads to the instability of embedded projects, and often cause delays in the projects. This will lead to the bias between platform's performance and customer requirement and affect product sales. Therefore, it is of great significance to construct the index system principle of requirement risk based on the theory of project requirement and the knowledge of embedded project.

\section{EMBEDDED SYSTEM}

In a broad sense, embedded system is any dedicated hardware or software system with a microprocessor [5]. Its core microprocessor has three types, namely digital signal processor (DSP), microcontroller (MCU) and embedded microprocessor (MPU).

In the narrow sense, the embedded system should adopt MPU to form an independent system, have its own operating system and has certain functions, the microprocessor of it must be at least 32 bits. Table 1 shows two embedded structural models.

Because of the flexibility and diversity of embedded systems, there is no uniform standard at all levels in Table 1 in other words, each individual system may be different. Therefore, the requirements for software designers would be high. On the one hand, it is necessary to combine the hardware in the software design. On the other hand, the software needs to be carried out on the hardware platform, both extend the development time of the system. To solve these problems, the designer should design a uniform interface function at the interface of the application and driving layer and shorten the development time. 
TABLE 1. two embedded structural models.

\begin{tabular}{|c|c|}
\hline & Application \\
\hline Application & API \\
\hline \multirow{2}{*}{ Driver/BSP } & OS \\
& HAL \\
\hline Hardware & Hardware \\
\hline Without OS & With OS \\
\hline
\end{tabular}

\section{REQUIREMENT RISK ASSESSMENT.}

To design a complete embedded system platform, we should not only consider the functional specification, but also consider the performance and cost and so on. Otherwise, it may end in a failed project. Therefore, prior to development, it is very necessary to learn some system techniques to make the development process smoother.

The design or selection of hardware platform plays an important role in the success of the project. Bad design or selection of hardware platform can lead to difficulties and delays in project development. The requirements of system are hard to describe, which lead to potential risks in requirement analysis. The risk comes from two main sources: the environment a $\mathrm{d}$ the requirement itself. This factor may lead to requirement risks:

The requirement provider lacks professional skills

Unclear requirement

Constantly changing requirements.

Considering the characteristics of the embedded system, the project requirements may have the following problems. First, the client can't express their requirements clearly, mainly on the method to achieve the function, and they have not described the specific functions in detail. Second, the cooperation between the two sides is not deep enough, sometimes they put the system, which is just slightly changed from the original product, in to produce without further debugging. Third, the requirement is uncompleted. The embedded system platform is a platform combined hardware with software. The contact between them may be missing, it requires developers to check the development process, build the structure, and then make the specification of requirement; Fourth, have not spend enough time to clarify the requirement. In the process of project development, we should devote enough time to clarify the requirements. However, in practice, the design company usually focuses on aspect like what functions the system has, to complete the project as soon as possible, and the time invested in the market for user control is very limited. It is very important to clarify the requirements with the clients. Figure 1 is the work flow of requirement deciding.

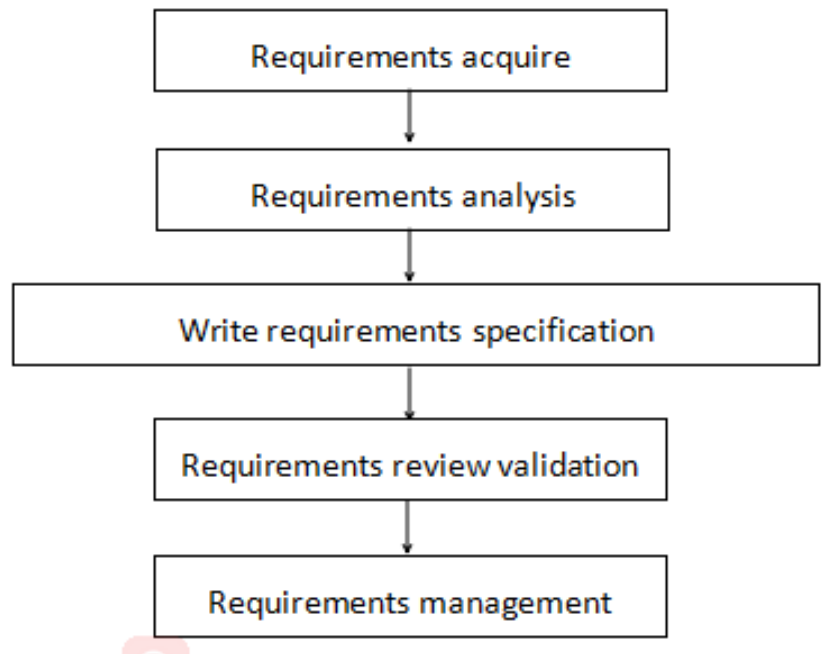

FIGURE 1. work flow of requirement deciding 


\section{INDICATOR SYSTEM PRINCIPLE}

Risk assessment [6] is the basis of risk management, including methodology and evaluation criteria [7]. The foundation of the evaluation standard is the safety assessment methodology, which is impartiality and the scientific. it has great significance to the risk assessment of embedded project. There are many methods of evaluation of indicator system [9], mainly including expert meeting analysis method [10], engineering analogy method, Delphi method and questionnaire method, etc.

To solve the problem, the principle of constructing the index system mainly includes the following aspects:

(1) Importance principle.

There are many principles to measure the risk of requirement, and the focus is different, so when selecting indicators, it can be divided into two levels: specificity and universality. In terms of specificity, it needs to choose representative and outstanding ones, which are specific indicators for embedded projects. We should pay attention to the relation and difference between indicators and avoid duplication or mutual inclusion between indicators to ensure that the project has a focus on risk assessment and can solve problems in a timely manner.

(2) Comprehensive principles.

This system must be able to objectively reflect the characteristics of all project requirements, evaluate the risk of the project requirements all cases from different aspects, and ensure authenticity and comprehensive risk assessment requirements.

(3) Consistency principle.

Project has different needs and ways, then the evaluation index system should be with the idea for product planning and users, to meet the common expectations, then expected and then pick out the unified evaluation index. The requirement index can fully utilize the platform to make the best effect and make the embedded products more competitive.

(4) Principle of independence.

When the index system is established, the indexes of the same level should be independent from each other. We should avoid overlapping, repetition and the correlation between indicators.

(5) Measurable principles.

The selected index system should be simple and easy to analyze, and it is better to use data calculation to show the accuracy and reliability of the products, which is conducive to the increase of product sales.

(6) Principle of universality.

The evaluation system should reflect all problems in an objective and comprehensive manner, must have the commonality, avoid the occurrence of small probability events, and must highlight the advantages of the product.

(7) Combining quantitative and qualitative indicators.

Quantitative index is more direct and specific. Using quantitative index in the evaluation, the result will be described clearer. however, not all the indicators can be quantified, so we need to design some specific indicators to feedback the problem, such as qualitative index. In contrast, qualitative indicators have much more information than quantitative ones, and the results are more comprehensive.

\section{OUTLOOK}

Embedded project management must be scientific and reasonable, which is the direction of industry development. The requirement risk assessment is the initial stage of the development of embedded project management and even plays a crucial role in the success or failure of the project. A good evaluation method can effectively manage the embedded project in the requirement risk, improve the management level of the project, increase the success rate of the project and make the embedded products serve the people in a better way.

\section{ACKNOWLEDGMENTS}

Philosophy and Social Sciences Research Project of Colleges and Universities in Jiangsu Province: Development and Implementation of Accurate and Intelligent Push System for College students' Abnormal Behaviors based on Big Data Hadoop Platform (No.2016SJB710095). 


\section{REFERENCES}

1. Mohammad Saniee Abarleh, Jafar Habibi, Zevnab Rarzegar, Muna Sergi. A parallel genetic local search algorithm for intrusion detection in computer networks[J]. Engineering Applications of Artificial Intelligence, 2007,20(8):1058-1069.

2. Luo L, Lan Y C, Tang Q. Corporate incentives to disclose carbon information: evidence from the CDP Global 500 Report[J]. Journal of International Financial Management\&Accounting.2012, 23(2): 93-120.

3. M an Esteve, J P Katoen, V Y Nguyen, et al. Formal correctness, safety, dependability, and performance analysis of a satellite[A]; Proceedings of the Proceedings of the 34th International Conference on Software Engineering[C], 2012, IEEE Press: 1022-1031.

4. Fan Xiao Juan. Quantitative evaluation of social stability risk in major engineering projects -- a case study of a mine and coal preparation plant $[\mathrm{J}]$. Engineering research, engineering in interdisciplinary vision, 2018, (01): 9898.

5. C Andre, F mallet. Description and verification of the time requirements of CCSL and esterel[A]; Journal of ACM SIGPLAN/SIGBED conference language, compiler and tools for embedded system [C],2009, ACM: 167167.

6. Teng Ying, he Xuejuan, Ni Debing. Risk assessment of private equity investment projects based on analytic hierarchy process and fuzzy comprehensive evaluation - a case study of 3 private equity investment projects [J]. Journal of electronic science and technology university (social science edition),2017,19(5): 67-73.

7. J Hasina. Describe and assess the availability requirements of the early stages of system development [J]. Software and system modeling, 2015,14(4):1455-1479.

8. Wang Chao, Jiang Ping, Wang Xiaoxing. Based on the research on the risk assessment of intelligent transportation project based on the fuzzy comprehensive evaluation method, taking the intelligent transportation project of the company as an example [J]. Journal of Hunan engineering institute (social science edition),2016,26(02):10 to 16 .

9. Zhu Zhengwei, Wang Qiong, Guo Xuesong. Analysis of social stability risks in engineering projects - analysis of factors based on public "risk - benefit" perception perspective [J]. Journal of xi 'a Jiao tong university (social science edition),2016, (03): 61-68.

10. L Dou,Q Liu,Z Yang, et al. A Situation Calculus-based semantic framework for UML[A]; Proceedings of the Communications and Information Technology (ICCIT),2012 International Conference on[C], 2012, IEEE: 7379. 